\title{
Novel Approach to Rhinosporidiosis
}

\author{
${ }^{1}$ Rajeshwary Aroor, ${ }^{2}$ Madhukar Muniswamy Gowda, ${ }^{3}$ Vadisha Srinivas Bhat, ${ }^{4}$ Satheesh Kumar Bhandary
}

\begin{abstract}
Rhinosporidiosis is a chronic enigmatic disease affecting mainly the upper aerodigestive tract and is notorious for frequent recurrences as high as $70 \%$. Higher incidences of recurrence are mainly due to nonendoscopic excision with resultant residual disease leading to seedling of the disease.
\end{abstract}

Objectives: The aim of this study is to highlight the factors responsible for recurrence and to follow the clinical course to ensure against recurrence of rhinosporidiosis. External surgery in rhinosporidiosis should be avoided for the fear of implantation. With the advent of endoscopes and Lasers allow the surgeon to complete excision of the lesion with cauterization of the base.

Materials and methods: A retrospective study of thirty two cases of rhinosporidiosis presented to us over a period of 10 years from 2002 to 2012 . Out of which 14 cases presented to us for the first time and 18 cases were recurrent cases. The commonest age group of presentation was between 20 and 30 years in patients presenting for the first time and in recurrent cases the commonest age of presentation was between 50 and 60 years of age. Majority of patients were males with male to female ratio of 9:1. All patients underwent endoscopic excision, electro cautery was used in 30 patients and Laser was used in 2 patients.

Results: Fourteen cases did not have any recurrence and in the 18 cases of recurrent cases three of them again had a recurrence where electro cautery was used to cauterize the base.

Conclusion: Endoscopic excision of rhinosporidiosis has a better result over nonendoscopic approaches with lesser recurrence rate. Our results show that use of endoscopes in patients who presented for the first time have a better results as the mucosa of recurrence patients have already been insulted due to previous surgeries.

Keywords: Rhinosporidiosis, Endoscopic excision, Electrocautery.

How to cite this article: Aroor R, Gowda MM, Bhat VS, Bhandary SK. Novel Approach to Rhinosporidiosis. Int J Otorhinolaryngol Clin 2014;6(2):55-57.

Source of support: Nil

Conflict of interest: None

\section{INTRODUCTION}

Rhinosporidiosis is a chronic infection commonly affecting the mucous membrane of nose and nasopharynx. Occasionally

\footnotetext{
${ }^{1,4}$ Professor, ${ }^{2}$ Resident, ${ }^{3}$ Associate Professor

${ }^{1-4}$ Department of ENT, KS Hegde Medical Academy, Mangalore Karnataka, India
}

Corresponding Author: Rajeshwary Aroor, Professor, Department of ENT, KS Hegde Medical Academy, Mangalore, Karnataka, India Phone: 08242204474, e-mail: rajeshwarisomayaji@gmail.com conjunctiva, lips, palate, larynx, trachea, bone, rectum and external genitalia are also can be involved. The disease is endemic in India and Sri Lanka but occurs sporadically in other parts of the world due to immigration and wide spread travel. ${ }^{1}$ The causative organism, rhinosporidiosis Seeberi had first been regarded as a protozoan by Malbran its discoverer in 1892, as a protozoan by Seeber who first published a description of the pathogen and then as a phycomecets by Ashworth in $1923 .{ }^{2}$ Herr et al, reclassified the organism as a member of protoctistan mesomycetozoa based on ribosomal DNA. $^{3}$

Rhinosporidiosis is notorious for its high rate of recurrence. Literature review suggests a residual or recurrence rate between 10 and $70 \%$. However, most reports show an incidence on an average of $10 \%$ related to incomplete excision. ${ }^{4}$ It is generally felt that external surgery in rhinosporidiosis should be avoided for the fear of implantation and inoculation of spores leading to more raw area exposed for further recurrences. ${ }^{5}$ It is surprising and sad that articles supporting external approach are getting published in English literature other than otorhinolaryngology journal (reference - a rare case of disseminated rhinosporidiosis highlighting need of specific management protocol: Journal of Oral Maxillofacial Surgery 2013;25:61-64).

Here, we report thirty-two cases of rhinosporidiosis managed by endoscopic excision and cauterization of the bases using electro cautery and Diode Laser. We feel that health education along with careful handling of the mass reduces the recurrence rate.

\section{MATERIALS AND METHODS}

This is a retrospective analysis conducted in our institution for a period of 10 years from 2002 to 2012 . A total no. of 32 cases of rhinosporidiosis were evaluated, out of 32 cases 29 were males $(91 \%)$ and 3 were females $(9 \%)$, with a higher male preponderance $(91 \%)$. The age of presentation was ranging from 11 to 70 years, majority between 50 and 70 years of age (37\%). Out of the thirty two cases 14 cases presented to us for the first time and 18 cases were recurrent cases previously treated elsewhere (Table 1). Majority of them gave a history of bathing in rivers or ponds that were used by their cattle. A detailed history including the age, sex, and duration of symptoms, personal habits and area of residence were noted. Particular focus was given to geographic distribution, the bathing habits, mode of 
Table 1: Distribution of cases

\begin{tabular}{llllll}
\hline Age & Male & Female & Total & New cases & $\begin{array}{l}\text { Recurrent } \\
\text { cases }\end{array}$ \\
\hline $0-20$ & 2 & - & 2 & 2 & - \\
$21-30$ & 7 & - & $7(22 \%)$ & 3 & 4 \\
$31-40$ & 4 & 1 & $5(16 \%)$ & 1 & 4 \\
$41-50$ & 6 & - & $6(19 \%)$ & 3 & 3 \\
$51-70$ & 10 & 2 & $12(37 \%)$ & 5 & 7 \\
\hline
\end{tabular}

previous surgery in recurrent cases. Clinical examination and diagnostic nasal endoscopic findings and modality of treatments were noted in all patients. Clinical findings on follow-up were noted with specific reference to recurrence.

All patients had undergone endoscopic excision of the mass followed by cauterization of the base. Electrocautery was used to cauterise the base and in two patients a diode Laser was used. All the fresh cases did not have any recurrence and in the recurrent rhinosporidiosis three of them again had a recurrence out of which one patient had recurrence in the nasopharynx which was again managed with endoscopic excision. Other 2 patients gave a history of multiple surgeries in the past (both had a history of 5 surgeries in the past). One patient had presented to us with pulmonary involvement with cutaneous lesions along with nasal lesion, patient refused for bronchoscopy and Laser excision and refused treatment. Patients in whom diode Laser was used did not have any recurrence.

\section{DISCUSSION}

Rhinosporidiosis is a chronic enigmatic disease commonly affecting the mucous membrane of nose and nasopharynx with multisystem involvement. It is endemic in India and Sri Lanka, but occurs sporadically in other parts of the world. In our study except one patient all other patients were from Kerala.

Literature review has suggested that rhinosporidiosis can occur as early as 3 years old with maximum incidence between 20 and 30 years with higher incidence in males $3: 1{ }^{4}$ In our study the earliest age of presentation was 11 years old and the incidence of fresh cases of rhinosporidiosis was between 20 and 40 years of age. However in our study maximum number of recurrent rhinosporidiosis cases were between 50 and 70 years of age. The male to female ratio was higher 9:1 as compared to 3:1 according to other the literature, this may be due to the fact that males more typically have jobs related to land and back water and also relating to bathing in ponds. Literature review suggests a residual or recurrence rate between 10 and $70 \%$. However most of the reports show recurrence rate of $10 \%$ related to incomplete excision of the mass. ${ }^{4}$ High recurrent cases of rhinosporidiosis presented to us in our study were probably due to lack of free availability of endoscopes in early nineties in peripheral health center and also lack of experience in endoscopic surgeries.

Several trials have shown that the most successful treatment with the lowest incidence of recurrence is endoscopic excision of the mass and cauterization of the base of the lesion. ${ }^{4-7}$ Mohan Kameshwaran had used KTP-532 Laser for rhinosporidiosis. According to him KTP-532 Laser is a superior tool as compared to diathermy excision. It enables the surgeon to obtain a better clearance margin with improved visibility and also decreases the total amount of blood loss. ${ }^{8}$ The use of endoscopes give better illumination and helps in removing the entire mass which cannot be seen by anterior rhinoscopy or conventional surgery and also helps in limited manipulation resulting in least resection of normal surrounding tissue. The physical contact of the tissue with Laser is less and hence the chances of seedling are reduced. Our experience with Laser is very limited. Since we have used Diode Laser we could not identify major differences between electro cautery and Laser in cauterization of base of rhinosporidiosis. Some authors have proposed a medical therapy with dapsone, ${ }^{9}$ but the results are inconclusive. Antimicrobial therapy is ineffective and the disease may recur after months or years. At present many cases of rhinosporidiosis are however unethically mismanaged by surgeons other than otorhinolaryngologist who deal with head and neck tumors. Otorhinolaryngologists in India are well aware of rhinosporidiosis and its management. It is unfortunate that very few articles about this enigmatic disease have been published in English literature and most of the published articles are regarding the clinical profile, microbiological profile and pathophysiological course. In the era of advents in endoscopes and Lasers, it is surprising that very few articles stress on endoscopic removal and Laser assisted excision which is the gold standard modality of treatment for rhinosporidiosis. We believe that high recurrence rate in earlier years were probably due to non-endoscopic removal of the masses thereby exposing more area for implantation and inoculation of the spores. With the recent advances in endoscopes and advents in Lasers it is advisable that all cases of rhinosporidiosis should be dealt accordingly to avoid recurrences. We believe that low recurrence in our study probably due to health education given to the patient regarding disease process of rhinosporidiosis, its transmission and prevention. These patients are strictly instructed not to take bath in the ponds. It is surprising that none of our patients other family members were suffering from rhinosporidiosis though other family members take bath in the same ponds. 
We also noted that even the spouses were not suffering from the disease. This indicates that some unknown host factor is responsible for rhinosporidiosis.

\section{CONCLUSION}

Nasal endoscopes provides better illumination to localize the site of origin ensuring complete removal of the rhinosporidiosis. Laser provides adequate cauterization with god hemostasis. The chances of seedling are reduced thereby minimising the complications and recurrence. Hence, we conclude that with recent advances in endoscopes and Lasers. It is advisable that all cases of rhinosporidiosis should be dealt by the experienced otorhinolaryngologist thus avoiding recurrences in future and reducing the burden of multiple surgeries.

\section{REFERENCES}

1. Arsecularatne SN. Recent advances in rhinosporidiosis and rhinosporidium Seeberi. Indian J Med Microbiol 2002; 20(3):119-131.
2. Ashworth JH. Rhinosporidium Seeberi (Wernick 1903) with special reference to its sporulation and affinities. Trans Roy Soc Edin 1923:53:301-342.

3. Herr RA, Aielho L, Taylor JW, et al. Phylogenetic analysis rhinosporidiosis seeberi. J Clinical Microb 1999;37:2750-2754.

4. Bhandary S, Natesh V, Chettri S, Kumar A. Rhinosporidiosis. Analysis of cases presenting to a tertiary care hospital in Nepal. Int J Tropical Med 2012;8(1):1-1.

5. Goyal NK, Mungutwar V, Banjara H, Chandrakar AK, Sudarshan V. Reader's views lacrimal sac diverticulum due to rhinosporidiosis. Indian J Otolaryngology Head Neck Surg 2011;63(4):411.

6. Das A, Das AK. Endoscopic excision of recurrent rhinosporidiosis. MJAFI 2008;64(1):76-77.

7. Sonkhya N, Singhal P, Mishra P. Nasooropharyngeal rhinosporidiosis: endoscopic removal. IJOHNS 2005;57(4):345-346.

8. Kameshwaran M, Singhal P, Anand Kumar RS, Sathiya M, Raghunandhan S, Jacob. KTP-532 Laser in the management of rhinosporidiosis. IJOHNS 2005:57(4):298-300.

9. Nair KK. Clinical trial of diaminodiphenylsulfone (DDS) in nasal and nasopharyngeal rhinosporidiosis. Laryngoscope 1979;89(2):291-295. 